\title{
B|O The Natural History of OPA1-related Autosomal Dominant Optic Atrophy
}

Amy C Cohn, Carmel Toomes, Alex W Hewitt, Lisa S Kearns, Chris F Inglehearn, Jamie E Craig and David A Mackey

Br. J. Ophthalmol. published online 24 Jul 2008;

doi:10.1136/bjo.2007.134726

Updated information and services can be found at:

http://bjo.bmj.com/cgi/content/abstract/bjo.2007.134726v1

These include:

Rapid responses You can respond to this article at:

http://bjo.bmj.com/cgi/eletter-submit/bjo.2007.134726v1

Email alerting Receive free email alerts when new articles cite this article - sign up in the box at the service top right corner of the article

Notes

Online First contains unedited articles in manuscript form that have been peer reviewed and accepted for publication but have not yet appeared in the paper journal (edited, typeset versions may be posted when available prior to final publication). Online First articles are citable and establish publication priority; they are indexed by PubMed from initial publication. Citations to Online First articles must include the digital object identifier (DOIs) and date of initial publication.

To order reprints of this article go to:

http://journals.bmj.com/cgi/reprintform

To subscribe to British Journal of Ophthalmology go to:

http://journals.bmj.com/subscriptions/ 
The Natural History of OPA1-related Autosomal Dominant Optic Atrophy.

Amy C Cohn, ${ }^{1}$ Carmel Toomes, ${ }^{2}$ Alex W Hewitt, ${ }^{1,3}$ Lisa S Kearns, ${ }^{1}$

Chris F Inglehearn, ${ }^{2}$ Jamie E Craig, ${ }^{3}$ David A Mackey. ${ }^{1}$

${ }^{1}$ Centre for Eye Research Australia, University of Melbourne, Department of Ophthalmology, Royal Victorian Eye and Ear Hospital, Melbourne, Australia. ${ }^{2}$ Section of Ophthalmology and Neuroscience, Leeds Institute of Molecular Medicine, University of Leeds, Leeds, UK.

${ }^{3}$ Department of Ophthalmology, Flinders Medical Centre, Adelaide, Australia.

Address for correspondence and reprints:

Associate Professor David A Mackey

Centre for Eye Research Australia,

Department of Ophthalmology, University of Melbourne

32 Gisborne Street

East Melbourne, VIC 3002

Australia

Telephone: +61399298713

Fax: +61 399298164

Email: D.Mackey@utas.edu.au

\section{Licence for Publication:}

The Corresponding Author has the right to grant on behalf of all authors and does grant on behalf of all authors, an exclusive licence (or non exclusive for government employees) on a worldwide basis to the BMJ Publishing Group Ltd to permit this article (if accepted) to be published in BJO and any other BMJPGL products and sublicences such use and exploit all subsidiary rights, as set out in our licence (http://bjo.bmj.com/ifora/licence.pdf).

\section{Competing Interest:}

None declared.

\section{Key words:}

Kjer's optic atrophy, genetic eye diseases, disease history, disease progression.

Word Count: 2033 


\section{ABSTRACT}

PURPOSE: Autosomal Dominant Optic Atrophy (ADOA) is a genetically heterogenous disease, however a large proportion is accounted for by mutations in OPA1. The aim of this longitudinal study was to investigate disease progression in Australian ADOA patients with confirmed OPA1 mutations.

METHODS: Probands with characteristic clinical findings of ADOA were screened for OPA1 mutations, and relatives of identified mutation carriers were invited to participate. Disease progression was determined by sequential examination or using historical records over a mean of 9.6 years (range 1-42 years).

RESULTS: 158 OPA1 mutation carriers were identified in 11 ADOA pedigrees. 69 mutation carriers were available for longitudinal follow up. Using the right eye as the default, best-corrected visual acuity (BCVAR) remained unchanged (defined as visual acuity at or within one line of original measurement) in 43 patients (62\%). BCVAR worsened by 2 lines in 13 patients (19\%). BCVAR deteriorated in by more than 2 lines in 6 patients (9\%). $10 \%$ of patients had an improvement in visual acuity. Mean time to follow-up was 9.6 years with the mean visual acuity being 6/18 for both the initial and subsequent measurements. There was no statistical significance in the rate of BCVAR loss across different OPA1 mutations $(\mathrm{P}=0.55)$.

CONCLUSION: OPA1-related ADOA generally progresses slowly and functional visual acuity is usually maintained. Longitudinal disease studies are important for allowing appropriate counselling of patients and this study allows for the better understanding of the natural history of ADOA. 


\section{INTRODUCTION}

Autosomal Dominant Optic Atrophy (ADOA)(MIM \#165500) is characterised by bilateral visual loss, centrocecal or paracentral scotomas, tritanopia or generalised colour dyschromatopsia and optic disc pallor with or without pseudo-cupping.[1-4] Visual loss generally commences during the first decade of life and is usually progressive. There is a large degree of inter and intra-familial phenotypic heterogeneity, making the accurate counselling of patients on likely disease severity difficult. $[5,6]$ The original descriptions, ADOA suggested full penetrance, however the identification of mutations in the OPA1 gene have allowed for more accurate phenotypic evaluation.[2, 710]

Reports quantifying disease progression serve as a useful resource for clinicians counselling patients. However, the rate of progression and final visual outcome of ADOA is difficult to predict and there are few studies addressing this specifically.[2, 8, 11] The purpose of this longitudinal study was to determine the severity and rate of disease progression in Australian ADOA patients with an OPA1 mutation.

\section{MATERIALS AND METHODS Subject Recruitment}

ADOA patients were recruited from South-eastern Australian. This study conformed to the tenets of the Helsinki Declaration and informed consent was obtained from all participants or their respective guardians when aged less than 18 years. Only participants confirmed with ADOA and had no other ocular pathology were recruited for this study.

\section{Mutation Screening}

Buccal swab or venipuncture specimens were obtained and DNA was extracted using either the Puragene (Gentra Systems, Minneapolis, USA) or Amersham Biosciences Nucleon BACC (Little Chalfont, United Kingdom) kits, respectively. OPA1 mutations were identified using single-strand conformational polymorphism and heteroduplex analysis (SSCP/HA) and then directly sequencing as previously described.[10]

\section{Clinical Examination}

Participants were examined using Snellen acuity charts or logMAR charts for best-corrected visual acuity (BCVA). Where the measurement was recorded in Snellen acuity, the reading was then converted to the logMAR equivalent for analysis. Farnsworth-Munsell 100 Hue test was used to test colour vision and visual fields were assessed with Humphrey 24-2 SITATM automated perimetry (Humphrey Field Analyser II, Zeiss-Humphrey, Dublin, California, USA). Stereoscopic optic disc photography was performed with a Nidek Stereo Fundus 3-Dx/F (Nidek, Gamagori, Japan) camera. Analysis of the optic cup area, vertical cup-to-disc ratio and neuro-retinal rim area was performed stereoscopically using custom software (StereoDx) with a Z-screen (StereoGraphics Corporation, Beverly Hills, California, USA).[12,13] The technical details of this system have been described elsewhere.[12,13] 
Longitudinal follow-up data were obtained from previous ophthalmic records or by direct clinical evaluation as part of this study. BCVA in the right eye (BCVAR) was used as the predominate outcome measure for disease progression. The Kruskal-Wallis test was used to compare the rate of change in BCVAR between pedigrees. The total number of subjects who remained at the same BCVAR level was compared using the $\chi^{2}$ test to that who had deteriorated and those who had improved.

Patients were categorised into the following groups: (1) no evidence of progression (defined as BCVAR either unchanged or within one logMAR visual acuity line); (2) loss of two logMAR equivalent lines in the right eye; (3) loss of more than two logMAR equivalent lines in the right eye; (4)

improvement in BCVAR by two or more logMAR visual acuity lines.

\section{RESULTS}

Of the 158 mutations carriers identified, longitudinal data were available for 69 subjects who at the most recent examination had a mean \pm SD age of 41.9 \pm 19.4 years (range 7 - 82 years)(Table 1 ). The mean \pm SD duration of followup was $9.6 \pm 7.9$ years (range 1 - 42 years). Two (2.9\%) subjects had followup of just one year. Eleven mutation carriers, with a mean BCVAR of 6/5.7 at the time of most recent measurement, reported being "asymptomatic" and therefore were unable to provide an accurate age of onset for reduced vision. Of the 58 (84\%) patients who were symptomatic, the mean age of onset of vision impairment was $10.2 \pm 10.1$ years (median 7 years). Across the whole study sample there was no statistical difference between initial BCVAR (mean 6/18; median 6/24; range 6/4.8 - HM) and BCVAR at follow-up (mean 6/18; median: 6/36, range 6/4 - HM). 
Table 1: Demographic Data of all patients in the longitudinal study of ADOA with confirmed OPA1 mutations.

\begin{tabular}{|c|c|c|c|c|c|c|c|c|c|}
\hline Family & Mutation & $\begin{array}{c}\mathrm{n} \text { family } \\
\text { members } \\
\text { screened }\end{array}$ & $\begin{array}{l}\text { n mutation } \\
\text { carriers (\%) }\end{array}$ & $\begin{array}{c}\text { n mutation } \\
\text { carriers } \\
\text { with } \\
\text { Iongitudinal } \\
\text { data }(\%) \\
\end{array}$ & $\begin{array}{c}\text { n symptomatic } \\
\text { patients with } \\
\text { longitudinal } \\
\text { data }(\%)\end{array}$ & $\begin{array}{c}\text { mean age } \\
\text { at final } \\
\text { review } \\
\text { (years) }\end{array}$ & $\begin{array}{c}\text { Mean } \\
\text { duration } \\
\text { of follow- } \\
\text { up } \\
\text { (years) }\end{array}$ & $\begin{array}{c}\text { mean } \\
\text { BCVAR at } \\
\text { initial } \\
\text { review }\end{array}$ & $\begin{array}{c}\text { mean } \\
\text { BCVAR at } \\
\text { final } \\
\text { review }\end{array}$ \\
\hline Vic-1 & Deletion of $O P A 1$ & 51 & $21(41.2)$ & $11(52.4)$ & $4(36.4)$ & 34.2 & 4 & $6 / 8.3$ & $6 / 8.0$ \\
\hline Vic-9 & 2708delTTAG & 4 & $3(75.0)$ & $2(66.7)$ & $2(100)$ & 30.5 & 6 & $6 / 42$ & $6 / 42$ \\
\hline Vic-12 & IVS9+1g>a & 7 & $5(71.4)$ & $5(100)$ & $5(100)$ & 45.4 & 18.2 & $6 / 42$ & $6 / 72$ \\
\hline Vic-13 & $1798 \mathrm{G}>\mathrm{T}$ & 18 & $11(61.1)$ & $3(27.3)$ & $3(100)$ & 50.6 & 13 & $6 / 16$ & $6 / 22$ \\
\hline Tas -4 & 2708delTTAG & 118 & $47(39.8)$ & $23(48.9)$ & $19(82.6)$ & 43.8 & 5.9 & $6 / 14.3$ & $6 / 14.1$ \\
\hline NSW-1 & $\begin{array}{c}937 A G>T A \& \\
1126 A>G\end{array}$ & 122 & $51(41.8)$ & $25(49.0)$ & $25(100)$ & 42.9 & 13.4 & $6 / 26.5$ & $6 / 30.8$ \\
\hline
\end{tabular}

Abbreviations: n, number; BCVAR, best-corrected visual acuity in the right eye. 
Analysing the right eye only, BCVAR remained unchanged in 43 patients $(62 \%)$ (Table 2$)$. Vision worsened by 2 logMAR lines in both eyes in 13 subjects (19\%) and by more than 2 lines in 6 subjects (9\%). Vision improved in 7 patients $(10 \%)$ (Figure 1$)$.

Table 2: Progression of visual loss in patients with ADOA and confirmed OPA1 mutations

\begin{tabular}{ll}
\hline & Right Eye only \\
\hline BCVA improved by $>2$ logMAR lines & $7(10 \%)$ \\
BCVA unchanged & $43(62 \%)$ \\
BCVA worsened by 2 logMAR lines & $13(19 \%)$ \\
BCVA worsened by $>2$ logMAR lines & $6(9 \%)$ \\
\hline
\end{tabular}

No statistically significant difference in the rate of visual loss between families harbouring different OPA1 mutations was identified $(p=0.55)$. Pooling of data across all pedigrees, revealed that in the subset of patients whose disease progressed $(n=19)$, mean BCVAR at initial measurement was $6 / 14.5$ and subsequently was $6 / 25.2$ with a mean time to follow up of 13.4 years. Those who did not progress (43 patients) had initial BCVAR of 6/15.7 and then $6 / 16.5$, with a mean time of 8.1 years to follow up.

We separated study participants into those aged 40 years or less $(n=35)$ and those over the age of 40 years $(n=34)$. Patients in the younger cohort had a mean age of 26.1 years and BCVAR of 6/12.9. Those in the older group had a mean age of 59.4 years and BCVAR of 6/23.3. There were significantly more people with a BCVA worse than 6/24 who were aged greater than 30 years compared to those aged less than 30 years $(p<0.001)$.

There were 32 patients for whom serial optic disc photographs were available for comparison (Figure 2). The mean duration of follow-up subjects with for optic disc photography was 6.7 years, and no quantitative change in neuroretinal rim loss was identified (data not shown).

\section{DISCUSSION}

ADOA has been reported to manifest during childhood, often as early as three to five years of age, as an insidious, mostly symmetrical, progressive loss of vision. The severity of visual loss is highly variable.[2, 8, 11] Over a ten year period we found that approximately one third of patients had deterioration in BCVA. However, $62 \%$ of patients had no change in visual acuity in at least one eye.

The range in final BCVA observed in this study, is consistent with other studies which have reported that some ADOA patients maintain adequate vision to meet driving standards (defined as visual acuity of $6 / 12$ or better) throughout their lifetime, while others are classified as legally blind (visual 
acuity less than 6/60) as early as the fourth decade of life.[1, 7, 11] Early clinical studies of families with ADOA by Kline and Glaser in 1979[4] and Hoyt in 1980[1] revealed a range of visual acuities from $6 / 6-6 / 7.5$ to $6 / 120$. In addition, the intergenerational disease expression varies greatly, and it is impossible to predict disease severity on the basis of other family members. $[1,6]$

The insidious onset of disease is well established, and in the initial reviews of ADOA families, most patients were unaware of reduction in vision prior to commencement of schooling or formal visual assessment, and therefore report the age of onset usually between four and six years of age. Moreover, up to a quarter of patients may be unaware of subtle visual disturbance at the time of diagnosis despite being cognisant of the familial nature of the disease.[4] In addition, in their review of the clinical features of ADOA, Johnston et al. reported that only $58 \%$ of genetically confirmed ADOA subjects in their study reported visual impairment before 11 years of age.[2] In our cohort, $84 \%$ of people were symptomatic and had a mean age of onset of visual deterioration at 10.2 years. They also described affected individuals who reported visual impairment beginning as late as 60 years of age.

ADOA patients generally exhibit a gradual progression of visual loss, with most but not all experiencing visual deterioration with increasing age. [2, 6,8 , 14] Unlike the clinical course of other inherited optic atrophies such as Leber's Hereditary Optic Neuropathy (LHON), it is very rare to have rapid visual deterioration in ADOA. Longitudinal [3] and cross-sectional studies [11] have shown disease progression with advancing age in most families. Long term follow-up (mean: 10 years) by Kjer showed that visual loss progressed in approximately $50 \%$ of his study cohort, however there was no disease severity quantification. Older patients overall had worse visual acuity that younger patients. $[3,8]$ This was also demonstrated in this study with our younger patients having a mean BCVAR of 6/12.9 and the older cohort with BCVAR of 6/23.3. Eliott et al. studied 20 ADOA patients to quantify the rate and severity of visual loss with time.[14] With a mean age at follow-up of 36 years (range: 9 - 72 years), Eliott and colleagues reported that the median visual acuity in ADOA patients decreased from 6/18 to 6/24 over a mean review period of 16 years (range: 5 - 40 years). They concluded that overall visual prognosis is relatively good in ADOA and that early diagnosis and appropriate counselling could minimise perceived visual disability. [14]

A significant limitation in any study requiring patients to nominate the age of onset of visual loss is recall reliability. Often previously undiagnosed mutation carriers would have their visual acuity assessed at school entry and the disease "discovered". This can lead to biases not only in recall but the true age of onset of visual loss.

Several studies have investigated test-retest variability in study subjects and the use of different visual acuity charts in the clinical setting.[15-17] Variance of less than one line improvement or deterioration on Snellen vision charts is interpreted as an acceptable level of "noise" especially in patients with ocular pathology. In our retrospective study, different testing centre and charts were 
used each time, thus reducing the ability to standardise the results. Although all testing would ideally be completed using standardised assessment charts under identical testing conditions, the merging of such data has been recognised as an acceptable method for ensuring comparable units for analyses.[18-20]

In addition to test retest noise, there is often an apparent improvement in vision in normal children as they get older and are able to conduct the visual acuity test with more confidence.[21] However, it is also possible that there may be a true recovery of vision in some ADOA patients as is observed in LHON.[22]

Unlike the documentation of visual acuity, optic disc photography is a recent addition to ophthalmic practice. Therefore, due to the short interval between serial photographs there was minimal discernable difference in most of the patients' disc appearances. This is in keeping with the slowly progressive nature of ADOA. With ongoing serial photography now part of routine ophthalmological practice we will be better able to document individual's optic disc changes.

In conclusion, our study identified a similar rate of visual loss in ADOA pedigrees compared to other longitudinal follow-up work. Interestingly the initial overall visual acuity was almost identical to the follow up mean visual acuity. As typified by a slowly progressive disease, visual acuity in approximately half the cohort remained unchanged. Clinicians can reassure prospective parents and patients that OPA1 mutation carriers often maintain reasonable, functionally useful vision late into their disease. 


\section{REFERENCES:}

1. Hoyt CS. Autosomal dominant optic atrophy. A spectrum of disability. Ophthalmology. 1980;87: 245-51.

2. Johnston RL, Seller MJ, Behnam JT, et al. Dominant optic atrophy. Refining the clinical diagnostic criteria in light of genetic linkage studies. Ophthalmology. 1999;106: 123-8.

3. Kjer B, Eiberg H, Kjer $\mathrm{P}$, et al. Dominant optic atrophy mapped to chromosome $3 \mathrm{q}$ region. II. Clinical and epidemiological aspects. Acta Ophthalmol Scand 1996;74: 3-7.

4. Kline LB, Glaser JS. Dominant optic atrophy. The clinical profile. Arch Ophthalmol 1979;97: 1680-6.

5. Delettre C, Griffoin JM, Kaplan J, et al. Mutation spectrum and splicing variants in the OPA1 gene. Hum Genet 2001;109: 584-91.

6. Votruba M, Fitzke FW, Holder GE, et al. Clinical features in affected individuals from 21 pedigrees with dominant optic atrophy. Arch Ophthalmol 1998;116: 351-8.

7. Cohn AC, Toomes C, Potter C, et al. Autosomal dominant optic atrophy: penetrance and expressivity in patients with OPA1 mutations. Am J Ophthalmol 2007;143: 656-62.

8. Kjer B. Infantile Optic Atrophy with Dominant Mode of Inheritance: A Clinical and Genetic Study of 19 Danish Families. Acta Ophthalmol Scand 1959;Supplement 54: 9-146.

9. Thiselton DL, Alexander C, Taanman JW, et al. A comprehensive survey of mutations in the OPA1 gene in patients with autosomal dominant optic atrophy. Invest Ophthalmol Vis Sci 2002;43: 1715-24.

10. Toomes C, Marchbank NJ, Mackey DA, et al. Spectrum, frequency and penetrance of OPA1 mutations in dominant optic atrophy. Hum Mol Genet 2001;10: 1369-78.

11. Votruba M, Moore AT, Bhattacharya SS. Clinical features, molecular genetics, and pathophysiology of dominant optic atrophy. J Med Genet 1998;35: 793800.

12. Morgan J, Sheen N, North R, et al. Discrimination of glaucomatous optic neuropathy by digital stereoscopic analysis. Ophthalmology 2005;112: 855862.

13. Morgan J, Sheen N, North R, et al. Digital imaging of the optic

nerve head: monoscopic and stereoscopic analysis. Br J Ophthalmol 2005;89: 879884.

14. Eliott M, Traboulsi E, Maumenee I. Visual prognosis in autosomal dominant optic atrophy (Kjer type). Am J Ophthalmol 1993;115: 360-7.

15. Arditi $A$, Cagenello $R$. On the statistical reliability of letter-chart visual acuity measurements. Invest Ophthalmol Vis Sci 1993;34: 120-9.

16. Bailey I, Lovie J. New design principles for visual acuity letter charts. Am J Optom Physiol Opt 1976;53: 740-45.

17. Ferris FL, 3rd, Kassoff $A$, Bresnick GH, et al. New visual acuity charts for clinical research. Am J Ophthalmol 1982;94: 91-6.

18. Holladay JT. Conversion of Snellen Acuity to logMAR visual acuity: ASCRS, 2004.

19. Hussain B, Saleh GM, Sivaprasad S. Changing from Snellen to LogMAR:debate or delay. Clin Experiment Ophthalmol 2006;34: 6-8. 
20. Moutray T, Williams M, Jackson A. Change of visual acuity recording methods in clinical studies across the years. Ophthalmologica 2008;222: 173-177.

21. Cotter SA, Tarczy-Honoch K, Wang Y. Visual Acuity testability in AfricanAmerican and Hispanic Children:The Multi-Ethnic Pediatric Eye Disease Study. Am J Ophthalmol 2007;144: 663-667.

22. Mackey DA, Howell, N. A variant form of Leber Hereditary Optic Neuropathy characterised by recovery of vision and multistep mitochondrial genetic etiology. Am J Hum Genet 1992;51: 1218-28. 


\section{Acknowledgments:}

We are grateful to Pfizer Australia for providing a research travel grant for ACC. This research was also supported by Glaucoma Australia, the Ophthalmic Research Institute of Australia, The Wellcome Trust, Yorkshire Eye Research and The Royal Society. CT is a Royal Society University Research Fellow, JEC is supported in part by an NHMRC Practitioner Fellowship and DAM is the recipient of a Pfizer Australia Research Fellowship.

\section{Competing Interest:}

None declared. 


\section{Figure Legends}

\section{Figure 1:}

The change in BCVA of the right eye of OPA1 mutation carrying patients from Australian ADOA pedigrees. a) patients with the 2708delTTAG OPA1 mutation (Tas4 ); b) compound heterozygous patients with the 937AG>TA and 1126A>G OPA1 mutations (NSW-1); c) patients with the complete deletion of OPA1 (Vic1); d) patients with the 2708delTTAG ( $\triangle$ Vic-9), 1798G $>$ T (o Vic-13) and the IVS9+1g >a mutation $(\bullet$ Vic-12).

\section{Figure 2:}

Longitudinal change in optic nerve head features of patients carrying OPA1 mutations. An asymptomatic carrier (Vic1-36) aged 39 with a BCVA of 6/6 (1a) and at age 43 with the same BCVA (1b); a mildly affected OPA1 mutation carrier (NSW1$51)$ at the age of 20 years with a BCVA of $6 / 9(2 a)$ and at the age of 29 years with a BCVA of 6/15 (2b); a moderately affected carrier (NSW1-18) at the age of 35 years with a BCVA of $6 / 18$ (3a) and aged 41 years with a BCVA of $6 / 36$ (3b); a severely affected carrier (Tas4-28) with a BCVA of 6/36 at the age of 29 (4a) and aged 34 years with a BCVA of $6 / 60(4 b)$. 


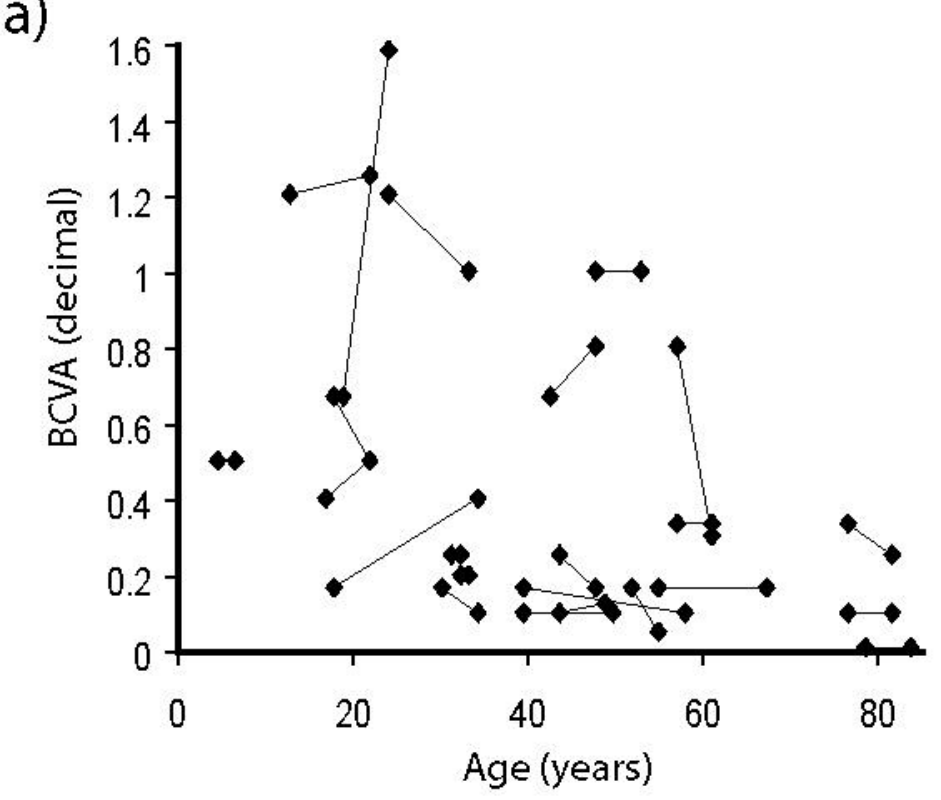

c)

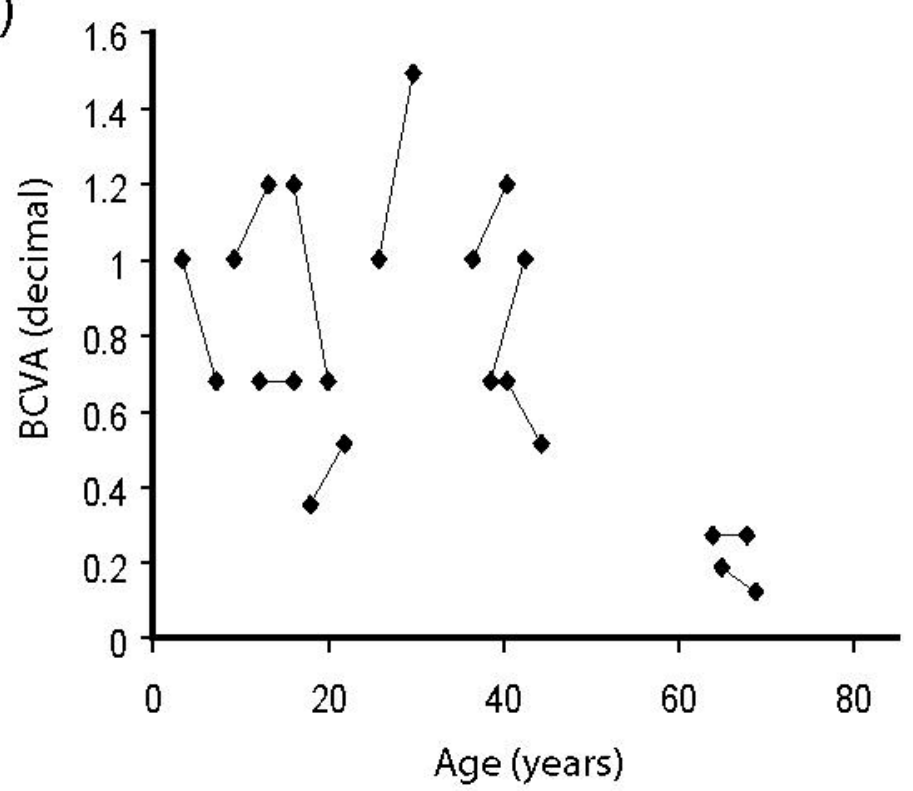

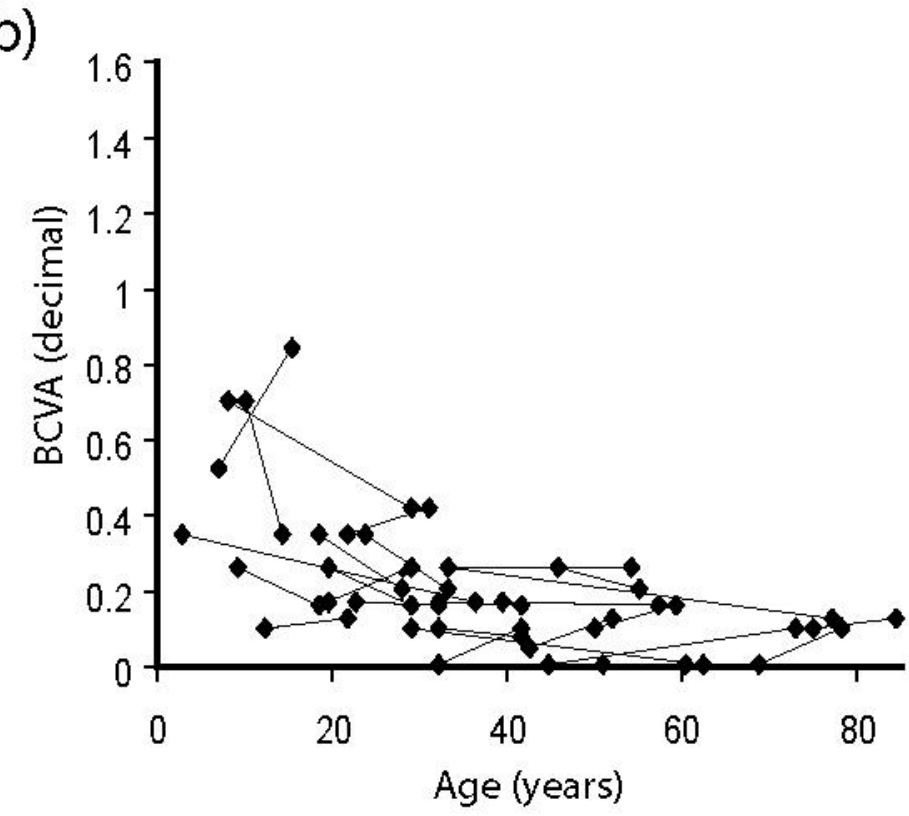

d)

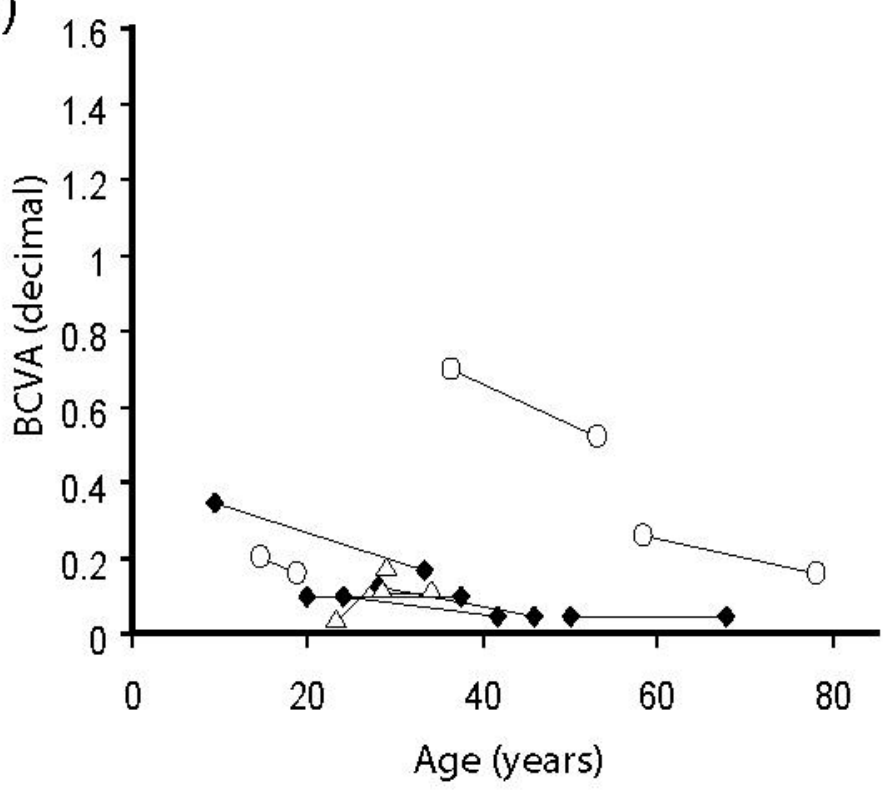


$1 a$

$2 a$

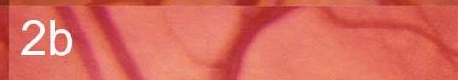

$3 a$
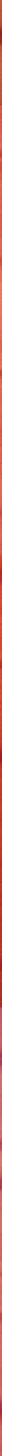

$3 b$

$4 a$

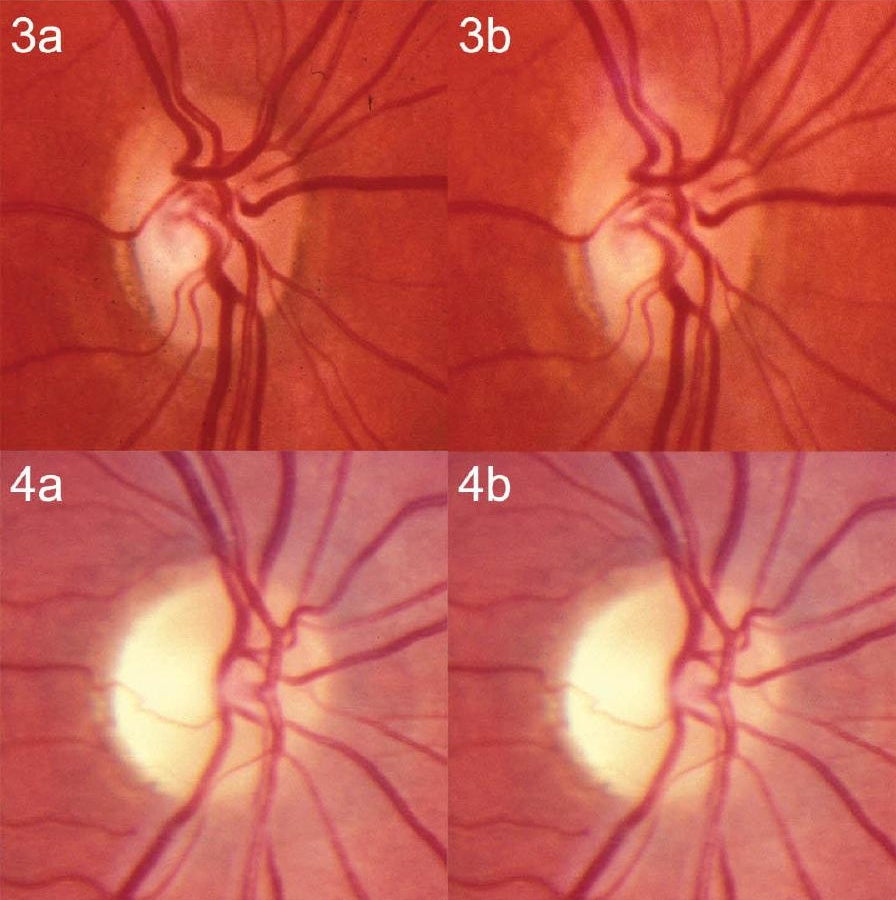

